\title{
The effect of higher-order dispersion on slow light propagation in photonic crystal waveguides
}

\author{
R.J.P. Engelen ${ }^{1}$, Y. Sugimoto ${ }^{2,3,4}$, Y. Watanabe ${ }^{4}$, J.P. Korterik ${ }^{5}$, \\ N. Ikeda ${ }^{2,3}$, N.F. van Hulst ${ }^{5,6}$, K. Asakawa ${ }^{2,4}$, L. Kuipers ${ }^{1,5}$ \\ ${ }^{1)}$ FOM Institute for Atomic and Molecular Physics (AMOLF), Kruislaan 407, 1098SJ \\ Amsterdam, The Netherlands \\ ${ }^{2)}$ The Femtosecond Technology Research Association (FESTA), Tsukuba, 300-2635, Japan \\ ${ }^{3)}$ National Institute of Advanced Industrial Science and Technology (AIST), \\ Tsukuba, 305-8561, Japan, at present \\ 4) TARA Center, University of Tsukuba, Tsukuba, 305-8577, Japan, at present \\ ${ }^{5)}$ Applied Optics Group, Department of Science \& Technology and MESA+ Institute for \\ Nanotechnology, University of Twente, P.O. Box 217, 7500AE Enschede, The Netherlands \\ ${ }^{6)}$ Institut de Ciències Fotòniques (ICFO), Av. Canal Olímpic, 08860 Castelldefels, \\ Barcelona, Spain, at present \\ engelen@amolf.nl
}

\begin{abstract}
We have studied the dispersion of ultrafast pulses in a photonic crystal waveguide as a function of optical frequency, in both experiment and theory. With phase-sensitive and time-resolved near-field microscopy, the light was probed inside the waveguide in a non-invasive manner. The effect of dispersion on the shape of the pulses was determined. As the optical frequency decreased, the group velocity decreased. Simultaneously, the measured pulses were broadened during propagation, due to an increase in group velocity dispersion. On top of that, the pulses exhibited a strong asymmetric distortion as the propagation distance increased. The asymmetry increased as the group velocity decreased. The asymmetry of the pulses is caused by a strong increase of higher-order dispersion. As the group velocity was reduced to $0.116(9) \cdot c$, we found group velocity dispersion of $-1.1(3) \cdot 10^{6} \mathrm{ps}^{2} / \mathrm{km}$ and third order dispersion of up to $1.1(4) \cdot 10^{5} \mathrm{ps}^{3} / \mathrm{km}$. We have modelled our interferometric measurements and included the full dispersion of the photonic crystal waveguide. Our mathematical model and the experimental findings showed a good correspondence. Our findings show that if the most commonly used slow light regime in photonic crystals is to be exploited, great care has to be taken about higher-order dispersion.
\end{abstract}

(C) 2006 Optical Society of America

OCIS codes: (160.3130) Integrated optics materials; (190.5530) Pulse propagation and solitons; (230.7370) Waveguides; (260.2030) Dispersion

\section{References and links}

1. See for example, Photonic Crystals and Light Localization in the 21st Century, in NATO Science Series, C.M. Soukoulis, ed. (Kluwer Academic, Dordrecht, The Netherlands, 2001)

2. S.G. Johnson, P.R. Villeneuve, S. Fan, J.D. Joannopoulos, "Linear waveguides in photonic crystal slabs," Phys. Rev. B 62, 8212-8222 (2000)

3. Y. Sugimoto, Y. Tanaka, N. Ikeda, Y. Nakamura, K. Asakawa, K. Inoue, "Low propagation loss of $0.76 \mathrm{~dB} / \mathrm{mm}$ in GaAs-based single-line-defect two-dimensional photonic crystal slab waveguides up to $1 \mathrm{~cm}$ in length," Opt. Express 12, 1090-1096 (2004) http://www.opticsexpress.org/abstract.cfm?URI=OPEX-12-6-1090 
4. E. Chow, S.Y. Lin, J.R. Wendt, S.G. Johnson, J.D. Joannopoulos, "Quantitative analysis of bending effiency in photonic crystal wavgeuide bends at $\lambda=1.55 \mu m$ wavelengths," Opt. Lett. 26, 286-288 (2001)

5. Y. Akahane, T. Asano, B.S. Song, S. Noda, "High-Q photonic nanocavity in a two-dimensional photonic crystal," Nature 425, 944-947 (2003)

6. A.Y. Petrov, M. Eich, "Zero dispersion at small group velocities in photonic crystal waveguides," Appl. Phys. Lett. 85, 4866-4868 (2004)

7. V.N. Astratov, R.M. Stevenson, I.S. Culshaw, D.M. Whittaker, M.S. Skolnick, T.F. Krauss, R.M. de la Rue, "Heavy photon dispersions in photonic crystal waveguides," Appl. Phys. Lett. 77, 178-180 (2000)

8. M. Notomi, K. Yamada, A. Shinya, J. Takahashi, C. Takahashi, I. Yokohama, "Extremely Large Group-Velocity Dispersion of Line-Defect Waveguides in Photonic Crystal Slabs," Phys. Rev. Lett. 87, 253902 (2001)

9. K. Inoue, N. Kawai, Y. Sugimoto, N. Ikeda, K. Asakawa, "Observation of small group velocity in twodimensional AlGaAs-based photonic crystal slabs,” Phys. Rev. B 65, 121308 (2002)

10. Y.A. Vlasov, M. O'Boyle, H.F. Hamann, S.J. McNab, "Active control of slow light on a chip with photonic crystal waveguides" Nature 438, 65-69 (2005)

11. for a review see M. Soljacic, J.D. Joannopoulos, "Enhancement of nonlinear effects using photonic crystals," Nature Mater. 3 211-219 (2004)

12. M. Notomi, A. Shinya, S. Mitsugi, G. Kira, E. Kuramochi, T. Tanabe, "Optical bistable switching action of $\mathrm{Si}$ high-Q photonic-crystal nanocavities," Opt. Express 13 2678-2687 (2005) http://www.opticsexpress.org/abstract.cfm?URI=OPEX-13-7-2678

13. A. Sugitatsu, T. Asano T, S. Noda, "Characterization of line-defect-waveguide lasers in two-dimensional photonic-crystal slabs," Appl. Phys. Lett. 84 5395-5397 (2004)

14. S.F. Mingaleev, Yu.S. Kivshar, R.A. Sammut, "Longrange interaction and nonlinear localized modes in photonic crystal waveguides," Phys. Rev. E 625777 (2000)

15. M.D. Rahn, A.M. Fox, M.S. Skolnick, T.F. Krauss, "Propagation of ultrashort nonlinear pulses through twodimensional AlGaAs high-contrast photonic crystal waveguides," J. Opt. Soc. Am. B 19, 716-721 (2002)

16. S. Yamada, Y. Watanabe, Y. Katayama, J.B. Cole, "Simulation of optical pulse propagation in a two-dimensional photonic crystal waveguide using a high accuracy finite-difference time-domain algorithm," J. Appl. Phys. 93, 1859-1864 (2003)

17. A. Imhof, W.L. Vos, R. Sprik, A. Lagendijk, "Large dispersive effects near the band edges of photonic crystals," Phys. Rev. Lett. 83, 2942-2945 (1999)

18. T. Asano, K. Kiyota, D. Kumamoto, B.S. Song, S. Noda, "Time-domain measurement of picosecond light-pulse propagation in a two-dimensional photonic crystal-slab waveguide," Appl. Phys. Lett. 84, 4690-4692 (2004)

19. H. Gersen, T.J. Karle, R.J.P. Engelen, W. Bogaerts, J.P. Korterik, N.F. van Hulst, T.F. Krauss, L. Kuipers, "Real space observation of ultraslow light in photonic crystal waveguides," Phys. Rev. Lett. 94, 073903 (2005)

20. M. Miyagi, S. Nishida, "Pulse spreading in a single-mode fiber due to third-order dispersion," Appl. Opt. 18, 678-682 (1979)

21. J. Khurgin, "Performance of nonlinear photonic crystal devices at high bit rates," Opt. Lett. 30, 643-645 (2005)

22. M.L.M. Balistreri, H. Gersen, J.P. Korterik, L. Kuipers, N.F. van Hulst, "Tracking femtosecond laser pulses in space and time," Science 294, 1080-1082 (2001)

23. S.G. Johnson, J.D. Joannopoulos, "Block-iterative frequency-domain methods for Maxwell's equations in a planewave basis," Opt. Express 8 $173-190$ (2001) http://www.opticsexpress.org/abstract.cfm?URI=OPEX-8-3-173

24. R.J.P. Engelen, T.J. Karle, H. Gersen, J.P. Korterik, T.F. Krauss, L. Kuipers, N.F. van Hulst, "Local probing of Bloch mode dispersion in a photonic crystal waveguide," Opt. Express 13, 4457-4464 (2005) http://www.opticsexpress.org/abstract.cfm?URI=OPEX-13-12-4457

25. Product information sheet, "Corning SMF-28e Optical Fiber, Product Information" (Corning Inc., 2005) http://corning.com/opticalfiber/products\%5F\%5Fapplications/products/smf\%5F28e.aspx

26. P.St.J. Russell, "Optics of Floquet-Bloch Waves in Dielectric Gratings," Appl. Phys. B 39, 231-246 (1986)

27. B. Lombardet, L.A. Dunbar, R. Ferrini, R. Houdre, "Fourier analysis of Bloch wave propagation in photonic crystals," J. Opt. Soc. Am. B 22, 1179-1190 (2005)

28. G.P. Agrawal, Nonlinear Fiber Optics (Academic Press, San Diego, Calif., 2001)

29. H. Gersen, J.P. Korterik, N.F. van Hulst, L. Kuipers, "Tracking ultrashort pulses through dispersive media: Experiment and theory" Phys. Rev. E 68, 026604 (2003)

30. M.L.M. Balistreri, A. Driessen, J.P. Korterik, L. Kuipers, N.F. van Hulst, "Quasi interference of perpendicularly polarized guided modes observed with a photon scanning tunneling microscope," Opt. Lett. 25, 637-639 (2000)

31. P. Sanchis, P. Bienstman, B. Luyssaert, R. Baets, J. Marti, "Analysis of butt coupling in photonic crystals," IEEE J. Quantum Electron. 40, 541-550 (2004)

32. H. Gersen, E.M.P.H. van Dijk, J.P. Korterik, N.F. van Hulst, L. Kuipers, "Phase mapping of ultrashort pulses in bimodal photonic structures: A window on local group velocity dispersion," Phys. Rev. E 70, 066609 (2004)

\#9936 - \$15.00 USD

(C) 2006 OSA
Received 13 December 2005; revised 13 February 2005; accepted 13 February 2006 20 February 2006 / Vol. 14, No. 4 / OPTICS EXPRESS 1659 


\section{Introduction}

Photonic crystals $(\mathrm{PhCs})$ are promising materials for the miniaturization of integrated optics and for the enhancement of light-matter interactions [1]. The periodic modulation of the refractive index in a PhC has a large influence on the optical dispersion relation. Particularly interesting is the existence of a photonic bandgap: a range of optical frequencies that are forbidden to propagate in the material. If a waveguide is embedded in a $\mathrm{PhC}[2,3]$, the light cannot escape the waveguide through the $\mathrm{PhC}$. This phenomenon can be used to create sharp waveguide bends and high-Q cavities [4,5]. In this way, a photonic bandgap can be exploited for the fabrication of integrated optical devices on the micron scale.

Photonic crystal waveguides (PhCWs) also exhibit interesting optical properties, since the dispersion relation of the light inside the waveguide is still influenced by the periodic nature of the surrounding crystal [6]. An interesting feature in both bulk PhCs and PhCWs is, that light will propagate with a very low group velocity at some specific optical frequency $[7,8,9,10]$. Hence, light-matter interaction is very strong and can be exploited for enhanced non-linear interactions in PhCWs [11]. Recently, all-optical switching in PhCs has been demonstrated [12] and PhCW lasing has been reported [13]. Also optical solitons have been proposed [14] and the nonlinear propagation of ultrafast pulses has been reported [15].

The group velocity is usually strongly dependent on frequency. This effect is quantified by the group velocity dispersion (GVD). Since every optical pulse has a certain spectral content, an optical pulse will experience a broadening due to the GVD. In PhCWs, this effect occurs at much shorter propagation lengths compared to conventional waveguides. Since the dispersive properties can be tailored by choosing the proper geometry, a tailored GVD can be exploited for pulse compression [16]. This so-called dispersion control of pulses in PhCWs is one of the promising applications of photonic crystals.

Recently, a number of studies have investigated the dispersive effect of $\mathrm{PhC}$ structures on pulse propagation $[17,18,19]$. Generally, these studies report on the reduction of the group velocity. Additionally, Asano et al. [18] also quantified enhanced group velocity dispersion in a PhCW. Enhanced GVD was also found in bulk 3D photonic crystals [17]. In this work, evidence was found of third order dispersion (TOD) [20], but not quantified. Aspects of third and higher-order dispersion have been discussed in theory in the context of slow light propagation in nonlinear photonic crystals [21].

For a detailed analysis of the dispersion of an optical pulse in a waveguide, one would ideally want to monitor the pulse shape as it propagates through a structure. Only then can the evolution of the pulse shape be evaluated as it propagates, and pulse distortion due coupling in and out of the sample therefore be excluded. Since out-of-plane radiation is absent for truly guided light, i.e. under the light line in a perfect structure, the study of the dispersion effects of a single, long structure as a function of position, would rely on scattering at defects, which is not desirable. Alternatively, structures of different length can be used, which has the disadvantage that the dispersion effects are studied in different waveguides. We circumvent these disadvantages by using a near-field approach. Near-field microscopy allows the local investigation of propagating light with a sub-wavelength resolution, while the structure under investigation is not disturbed [22].

Here we present the near-field probing of optical pulses as they travel through a photonic crystal waveguide. As we changed the optical frequency and in this way accessed slower propagating light, the dispersion of the pulses increases dramatically. The pulse shapes change from initially symmetric to broader and more asymmetric pulse envelopes, as the group velocity decreases. The measurements are reproduced by calculating the expected pulses shapes from the dispersion relation. Thus we demonstrate that higher-order dispersive effects play an important role in the propagation of light in photonic crystal waveguides. These higher-order dispersive

\#9936 - \$15.00 USD

(C) 2006 OSA
Received 13 December 2005; revised 13 February 2005; accepted 13 February 2006 20 February 2006 / Vol. 14, No. 4 / OPTICS EXPRESS 1660 
effects can be detrimental to many slow light applications and should be taken care of by proper dispersion engineering, by which method the disadvantage can be even turned into an advantage.

\section{Experimental aspects and modelling}

\subsection{Sample and experimental setup}

The samples used in the experiments were fabricated in epitaxial hetero-structures grown by molecular beam epitaxy. A 250 -nm-thick GaAs core layer was grown on top of a $2-\mu \mathrm{m}$-thick $\mathrm{Al}_{0.6} \mathrm{Ga}_{0.4} \mathrm{As}$ sacrificial layer on a GaAs substrate. A membrane-type photonic crystal structure was fabricated using high-resolution electron-beam lithography, dry etching, and selective wetetching techniques. The $2 \mathrm{D} \mathrm{PhC}$ thus consisted of a hexagonal array of air holes etched into a planar GaAs slab. The sacrificial layer was removed by an HF solution via the air holes. The lattice constant is $339 \mathrm{~nm}$ with air-holes of $204 \mathrm{~nm}$ in diameter. By leaving a single row of airholes unperforated, a so-called W1 waveguide was created. A section of the resulting structure is shown in Fig. 1.

The propagation of light in the $\mathrm{W} 1$ waveguide is described by the dispersion relation, shown in Fig. 2(a). The dispersion relation of the $\mathrm{W} 1$ waveguide was calculated by 3D plane wave expansion [23]. In the dispersion relation, we found two waveguide modes within the photonic crystal bandgap for TE polarization. These modes are denoted odd and even in Fig. 2. The even mode is largely underneath the light line and is therefore in principle lossless [3]. Near $\omega=0.254$ the even mode bends towards $k_{z}=\pi / a$, corresponding to a strong reduction of the group velocity $\left(v_{g}\right)$, since the $v_{g}$ is determined by the inverse of the slope of $k_{z}(\omega)$. Note that the 2D bandgap only exists for TE polarization. TM polarized light can either propagate through the crystal, or it can be confined to the waveguide by refractive index contrast [24].

In Fig. 2(b), the group velocity of the even TE-polarized mode is shown as a function of optical frequency. At high frequencies, $v_{g}$ is found te be $0.23 \cdot c$. As the frequency is reduced, the group velocity decreases to become zero at $\omega=0.253$. The group velocity dispersion (GVD) is defined by the second order derivative of the dispersion relation $\left(\beta_{2}=\mathrm{d}^{2} k / \mathrm{d} \omega^{2}\right)$. The frequency dependency of $\beta_{2}$ is shown in Fig. 2(c). At high frequencies, the GVD is in the order of $-10^{4}$ $\mathrm{ps}^{2} / \mathrm{km}$. As the frequency is reduced and the group velocity drops, the GVD increases by several

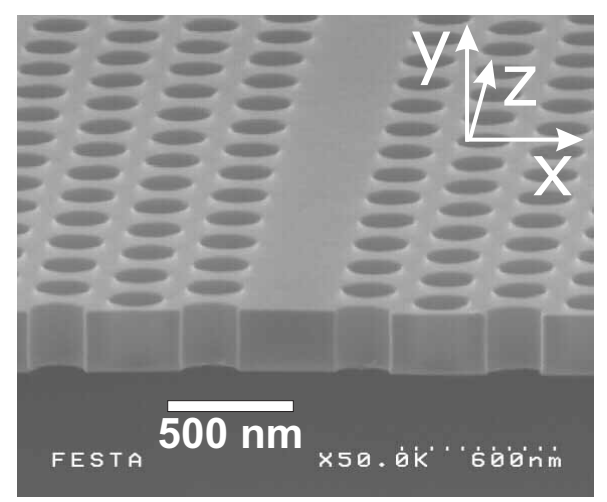

Fig. 1. Scanning electron microscopy image of the photonic crystal structure. The inset denotes the used cartesian coordinate system used in this paper. The $\mathrm{PhCW}$ is oriented in the $z$-direction. The $x$-direction is in the plane of the $\mathrm{PhC}$ and is perpendicular to $z$. The $y$-direction is perpendicular to the plane of the membrane.

\#9936 - \$15.00 USD

(C) 2006 OSA
Received 13 December 2005; revised 13 February 2005; accepted 13 February 2006 20 February 2006 / Vol. 14, No. 4 / OPTICS EXPRESS 1661 

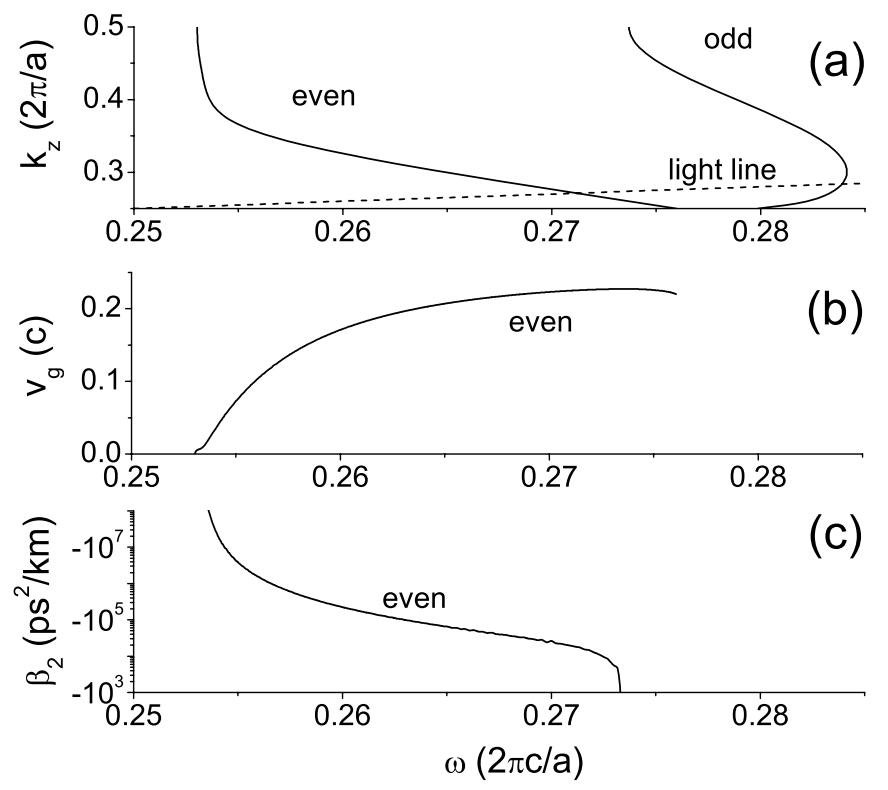

Fig. 2. (a) Portion of the dispersion relation of the $\mathrm{W} 1$ waveguide calculated by 3D plane wave expansion. In the $2 \mathrm{D}$ bandgap, two waveguide modes are allowed to propagate for TE polarization. They are denoted "even" and "odd" by their in-plane symmetry with respect to the waveguide. The light line $\left(\omega=c k_{z}\right)$ is represented by the dashed line. (b) Group velocity $\left(v_{g}\right)$ as a function of frequency, calculated by taking the derivative of $k(\omega)$. (c) The second order derivative of $k(\omega)$ gives the group velocity dispersion $\left(\beta_{2}=d^{2} k / d \omega^{2}\right)$. As the group velocity reduces around $\omega=0.254$, the GVD increases several orders of magnitude.

orders of magnitude. The GVD is many orders of magnitude larger than in fiber optics. For comparison, the GVD in an optical fiber is between -28 and $+8 \mathrm{ps}^{2} / \mathrm{km}$ [25]. Because, the GVD is also frequency-dependent, higher-order dispersive effects can also play a role in the propagation of light through PhCWs.

We monitored the broadening of femtosecond pulses as a function of propagation distance. Therefore we used laser light, obtained from a Ti:Sapphire-pumped optical parametric oscillator, that provided pulses with a tunable carrier wavelength between 1200 and $1350 \mathrm{~nm}$. The full width at half maximum (FWHM) of the pulse spectrum was $12 \mathrm{~nm}$, corresponding to a fourier limited pulse duration of $207 \mathrm{fs}$ (FWHM). These pulses were coupled into the PhCW by focussing the light onto the membrane facet with a microscope objective with a numerical aperture of 0.4 .

Figure 3(a) shows a focussed ion beam micrograph of a typical near-field fiber probe used in the experiments. The probe consisted of a tapered optical fiber, coated with an aluminum layer of approximately $100 \mathrm{~nm}$ thickness. The aluminum coating prevented stray light from entering the probe. At the tip apex, a sub-wavelength aperture was obtained by focussed ion beam milling. The aperture diameter was $230 \mathrm{~nm}$.

The probe was kept in the evanescent field of the propagating light and in this way, a minute fraction of the light was coupled into the fiber probe. A shear-force feedback mechanism kept the fiber probe at a constant $(\sim 10 \mathrm{~nm})$ distance above the membrane. We scanned the fiber probe 
(a)

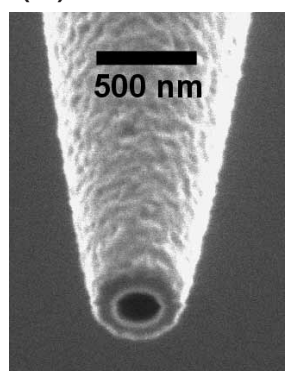

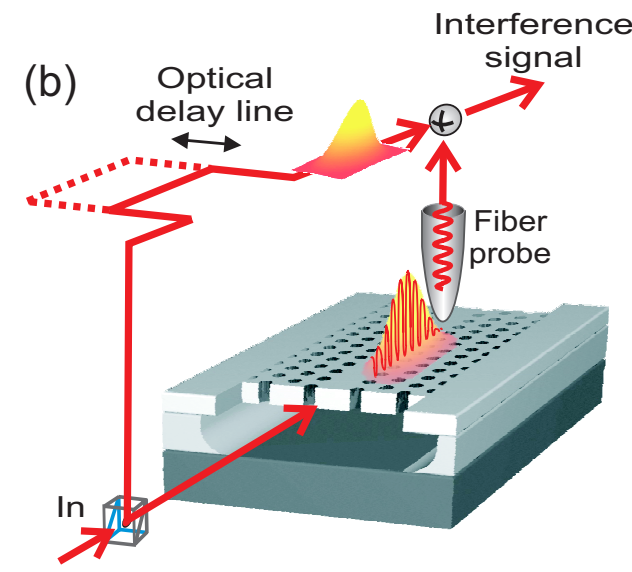

Fig. 3. (a) Focussed ion beam micrograph of the near-field probe, that consisted of a metalcoated tapered optical fiber. The typical aperture diameter is $200 \mathrm{~nm}$, for measurements in the infrared. The image was obtained after ion beam milling of the tip apex. View angle: $\sim 45 \mathrm{deg}$. (b) Schematic representation of the experimental setup. Pulsed laser light was coupled into the $\mathrm{PhCW}$. The fiber probe collected a fraction of the propagating light. This light was mixed interferometrically with a reference pulse from the same laser. Only the interference between the two pulses was detected. Two measurement modes were possible: either scanning the fiber probe over the surface with a fixed optical delay line, or scanning the delay line for a single probe position.

over the sample surface while using a heterodyne interferometric detection scheme, which yielded spatially-resolved, phase-sensitive and time-resolved information on the propagation light [22]. Figure 3(b) shows the heterodyne interferometric setup in a schematic representation. Before coupling the pulsed light into the $\mathrm{PhCW}$, the light was split into two parts. The light travelling through the sample and the fiber probe is called the signal branch. The other branch (reference), contained an optical delay line. Both the signal and reference light was mixed interferometrically and collected onto a detector. Using pulsed laser light, the interference will only be present when the pulses in both branched overlap in space and time at the mixing junction. In order to obtain temporal overlap, the optical path of the reference branch could be varied. Thus, our setup allowed two distinct measurement techniques: either scanning the fiber probe for a fixed delay line position (i.e. a fixed reference time) or scanning the delay line for a single probe position. Since both measurement schemes were used this investigation, these are explained in greater detail.

\subsection{Local heterodyne detection of pulse propagation}

In order to complement our experiments, we will compare the results with a model describing our measurement. Therefore, we will describe the propagation of light through a $\mathrm{PhCW}$ and model the detection of this light. The propagation of light through a medium with a period modulation of the dielectric constant, such as Bragg stacks or a photonic crystal, can be described with Bloch waves [26]. A Bloch wave can be decomposed into several plane wave components, each which a different wavevector $k_{m}$ :

$$
\tilde{E}(z, \omega)=\sum_{m} \tilde{E}_{m}(\omega) \exp \left(i k_{m}(\omega) z\right)
$$

\#9936 - \$15.00 USD

(C) 2006 OSA 


$$
\text { with } k_{m}(\omega)=k_{0}(\omega)+\frac{2 \pi}{a} m \text { and } m \in \mathbb{Z},
$$

where $a$ is the period of the lattice. (For simplicity, a one-dimensional lattice is considered.) The wavevectors of the plane waves that comprise a single Bloch wave are spaced $2 \pi / a$ apart in reciprocal space. The dispersion for all the higher-order Bloch harmonics, i.e. $m \neq 0$, is identical to that of $k_{0}(\omega)$. Calculating the dispersion relation (such as in Fig. 2(a)) in the first Brillouin zone suffices to represent the dispersive properties of the entire Bloch wave. For the effect on either the pulse shape in time or in space on length scales larger than a single unit cell we therefore consider only

$$
\tilde{E}(z, \omega)=\tilde{E}_{0}(\omega) \exp \left(i k_{0}(\omega) z\right),
$$

where $\tilde{E}_{0}$ and $k_{0}$ represent the amplitude of the electric field and the fundamental wavevector of the Bloch wave, respectively [26, 27]. Generally, $k_{0}(\omega)$ is approximated with a Taylor expansion:

$$
k_{0}(\omega)=\beta_{0}+\beta_{1}\left(\omega-\omega_{0}\right)+\frac{\beta_{2}}{2}\left(\omega-\omega_{0}\right)^{2}+\frac{\beta_{3}}{6}\left(\omega-\omega_{0}\right)^{3}+\ldots
$$

In this Taylor expansion, the coefficients $\beta_{i}$ represent the dispersive constants of a material. The wavevector equals $\beta_{0}, \beta_{1}$ is the inverse of the group velocity $\left(v_{g}\right), \beta_{2}$ and $\beta_{3}$ represent the GVD and the third order dispersion (TOD), respectively. The evolution of the optical electric field in space and time is obtained by Fourier transforming Eq. 3:

$$
E(z, t)=\mathscr{F}^{-1}\left\{\tilde{E}(\omega) e^{i k(\omega) z}\right\}
$$

A certain frequency bandwidth in $\tilde{E}(\omega)$ is therefore required to describe a short pulse in time. $\mathscr{F}^{-1}$ denotes the inverse Fourier Transform. For a medium without TOD $\left(\beta_{3}=0\right)$, this is analytically solvable for a Gaussian pulse [28, 29]. In this case, a Gaussian pulse propagates according to:

$$
E(z, t)=\frac{\tau_{p} E_{0}}{\sqrt{\tau_{p}^{2}-4 i z \beta_{2}}} \exp \left[i z \beta_{0}+i \omega_{0} t-\frac{\left(t-z \beta_{1}\right)^{2}}{\tau_{p}^{2}-4 i z \beta_{2}}\right] .
$$

In this equation, the Fourier-limited pulse duration is denoted $\tau_{p}$. Clearly, when the electric field is considered at one time $t$, a low group velocity (i.e. a high $\beta_{1}$ ) leads to a compression of the pulse in space. When the E-field at a specific point $(z)$ is evaluated, a $\beta_{2} \neq 0$ yields a broadened pulse. In addition, since the energy of the pulse is constant, the E-field amplitude is reduced. By evaluating the pulse broadening as a function of position $(z), \beta_{2}$ can be recovered.

If we consider the spatial envelope of a propagating pulse, we see that the accumulated dispersion differs. Assuming strong GVD, the trailing part of the pulse will have experienced less dispersion than the leading section of the pulse. Therefore, the accumulated GVD after propagating a distance $z$ is $z \beta_{2}$. When the quantity $z \beta_{2}$ is comparable to or larger than $\tau_{p}^{2}$, the envelope of the propagating pulse will be asymmetric in space. Please note that $\beta_{2}$ does not change the shape of the pulse envelope in time, it only changes its duration.

When third order dispersion is included, the pulse envelope can be approximated using the Airy function [20]. This implies, that for a specific $z$, the pulses are deformed asymmetrically in time. The temporal asymmetry of the pulses can be a measure for the TOD in the photonic material.

When also higher-order dispersive term are included, Eq. (5) is no longer analytically solvable. Then, Eq. (5) needs to be solved numerically. In such a numerical solution, the full dispersion relation $(k(\omega))$ is used as input. Hence the calculation is not limited by the accuracy of the Taylor expansion, since all dispersive orders are included, if present.

\#9936 - \$15.00 USD

(C) 2006 OSA 
In our experiment, we could investigate the pulse either in the temporal domain at a single position or measure a "snap-shot" of the pulse propagating in space for a fixed reference time. In the interferometric setup (see above), the optical frequency of the light in the reference branch was shifted by $40 \mathrm{kHz}$. As a result, whenever the light from the two branches interfere, we observed a $40 \mathrm{kHz}$ modulation in the detector output. The relative phase and amplitude could readily be measured using a lock-in amplifier (LIA) [30].

Here, we denote the electric field of the light in the reference branch as $E_{r e f}$. Compared to $E_{r e f}$, the E-field in the signal branch $\left(E_{\text {sig }}\right)$ differed in spectrum and amplitude. With this in mind, we calculated the detection of pulses in our interferometric set-up. The pulses in the reference branch were assumed to be Fourier-limited, while the pulses in the signal branch could be distorted by the dispersion in the sample. $E_{r e f}$ is therefore not dependent on position $z$, in contrast to $E_{\text {sig }}$. The light intensity on the detector can be described as:

$$
I_{\text {det }}(z, t)=\left|e^{i \Delta \omega(t-\tau)} E_{r e f}(t-\tau)+E_{s i g}(z, t)\right|^{2} .
$$

Note that the change in optical frequency of the reference branch is given by $\Delta \omega$. The delay time delay between the reference pulse signal pulse is denoted $\tau$. We can split Eq. (7) into a constant signal and an interference term:

$$
I_{\text {det }}(z, t)=\left|E_{\text {ref }}(t-\tau)\right|^{2}+\left|E_{\text {sig }}(z, t)\right|^{2}+2 \operatorname{Re}\left[E_{\text {ref }}^{*}(t-\tau) E_{\text {sig }}(z, t) e^{i(\Delta \omega t-\omega \tau-\Delta \omega \tau)}\right] .
$$

The first two $|E|^{2}$ terms are the constant detector signals, and the third term describes the interference. The real part of a function is denoted as "Re". With our Lock-In Amplifier (LIA), we detected only the interference term as only this term varied with $40 \mathrm{kHz}$, given by $\Delta \omega$. This signal was obtained in a characteristic time $\Delta t$. This characteristic time is given by the bandwidth of the detection system, and is therefore always much longer than the pulse duration $\left(\Delta t \gg \tau_{p}\right)$, the repetition rate and is also kept much longer than 1 divided by the $40 \mathrm{kHz}$ modulation. With this in mind, the output voltage of one of our LIA channels was proportional to:

$$
V_{L I A}(z, \tau) \propto \int_{\Delta t} 2 \operatorname{Re}\left[E_{r e f}^{*}(t-\tau) E_{s i g}(z, t)\right] d t .
$$

Note that the LIA output was only dependent on the delay time $\tau$ between reference and signal pulse and was not dependent on the time $t$. We can rewrite the above cross-correlation as a function multiplication in the spectral domain:

$$
V_{L I A}(z, \tau) \propto 2 \operatorname{Re}\left\{\mathscr{F}^{-1}\left[E_{\text {ref }}^{*}(\omega) E_{\text {sig }}(z, \omega)\right]\right\} .
$$

And if we assume propagating waves waves for $E_{\text {sig }}$, as in Eq. 2:

$$
V_{L I A}(z, \tau) \propto 2 \operatorname{Re}\left[\mathscr{F}^{-1}\left\{E_{\text {ref }}(\omega) E_{\text {sig }}(\omega) e^{i k z}\right\}\right] .
$$

We assume that the spectrum of the light in the signal branch was not necessarily equal to the spectrum of the light in the reference branch, due frequency-dependent group velocity in the waveguide. Therefore, not all frequencies would couple to the PhCW equally efficient and we assume that the coupling of the light to the waveguide obeyed Fresnel's law[31].

In the experiments, either $z$ or $\tau$ in Eq. (11) was fixed, while the other variable was varied. In this way, two measurement approaches were possible: a time-resolved measurement of the interference or a space-resolved measurement, experimentally corresponding to scanning either probe or delay time. In the first measurement scheme, the fiber probe was scanned over the sample. This resulted in the distribution of the interference as a function of position. We will refer to this measurement mode as the spatial interference distribution (SID) measurement and

\#9936 - \$15.00 USD

(C) 2006 OSA 


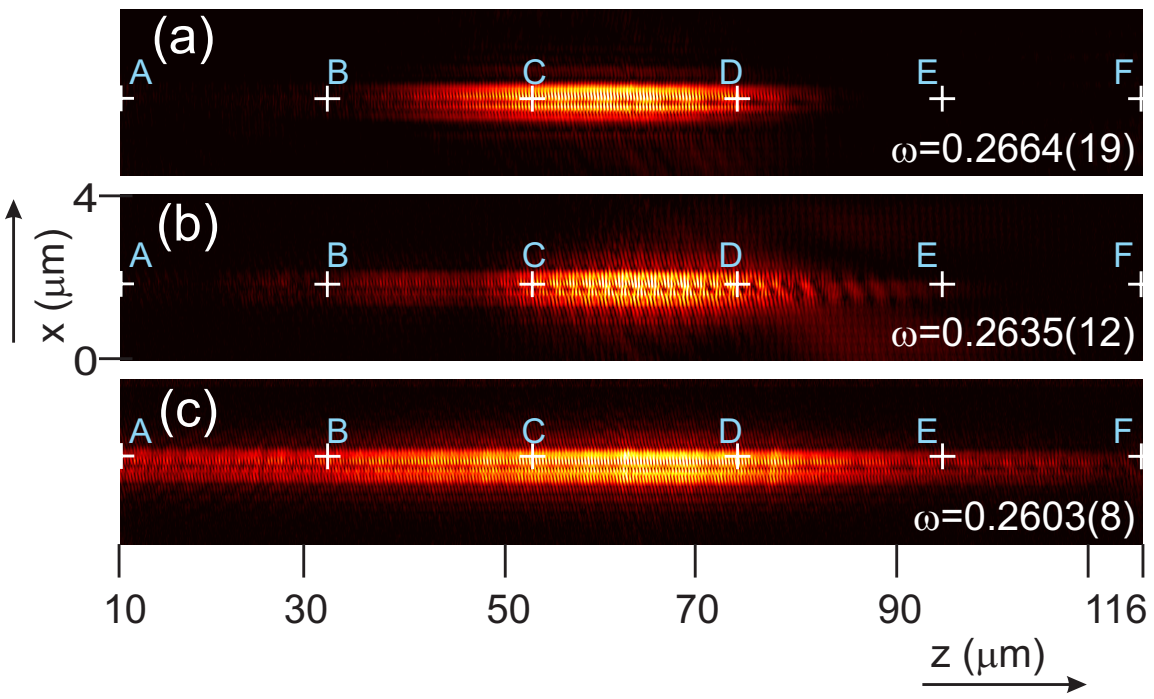

Fig. 4. Near-field optical measurement obtained by scanning the surface of the PhC sample. The false-color images represent the amplitude of optical interference. Measurements at normalized optical frequencies $0.2664,0.2635$ and 0.2603 are shown in (a), (b) and (c) respectively. Images size: $106 \mu \mathrm{m}$ x $4 \mu \mathrm{m}$. The crosses "A" up to "F"represent the positions were additional experiments were conducted.

it contains a combination of dispersion and pulse shape information. With this technique, spatial properties can be derived on the propagation of light, for example wavevectors and mode profiles [32].

In the second scheme, the probe was kept at a fixed position on the waveguide and the optical path length of the reference branch was varied. Now, a pulse cross-correlate (PXC) between the light in the two branches was obtained in time. With a PXC measurement, temporal information can be recovered, like optical frequency and pulse dispersion [17]. Obviously, overlapping conclusions can be drawn from either measurement (SID and PXC), like pulse dispersion. Nevertheless, the two methods complement each other. Only, by using both measurement approaches, the full picture can be obtained.

\section{Results}

\subsection{Near-field experiments}

Figure 4 shows the result of a SID measurement of the pulses in the W1 PhCW. At each scan position, the interference between the reference and signal (including sample) pulses is measured. Measurements are presented at the optical frequencies $0.2664,0.2635$ and 0.2603 in normalized units. The optical delay in the reference branch was chosen such that the maximum of the measured optical signal was found at approximately the same position. Because the group delay increased in these measurements with decreasing frequency, the delay $\tau$ was increased going from Fig. 4a to Fig. 4c. The pulses are found to be well confined to the waveguide in the lateral direction. At each frequency, the spatial distribution along the propagation direction is different. At the highest frequency $(\omega=0.2664)$, the pulse in the structure was least distorted by the dispersion and we find a smooth and short envelope of 25(2) $\mu \mathrm{m}$ (FWHM) in width. As the frequency was reduced, the measured pattern was elongated along the propagation direc-

\#9936 - \$15.00 USD

(C) 2006 OSA 

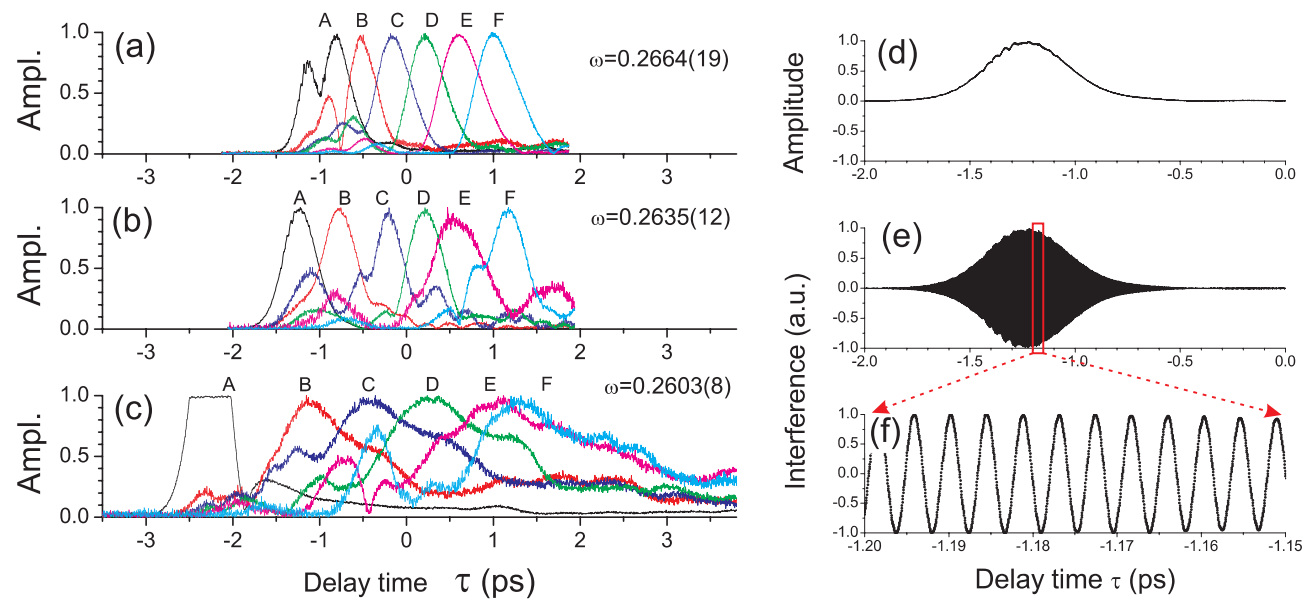

Fig. 5. (a-c) Cross-correlation functions of the pulses in reference and signal branch of the interferometric setup. At the optical frequencies 0.2664, 0.2635 and 0.2603 , the interferograms are measured at 6 equidistantly spaced points on the waveguide between $10 \mu \mathrm{m}$ and $116 \mu \mathrm{m}$ from the membrane facet. These measurement points are highlighted with crosses in Fig. 4. The measurement points "A"-"F" correspond to the points in Fig. 4, i.e. the interferogram "A" is measured at location "A". The interference amplitudes are normalized with respect to their maximum. In the first curve in (c), our detector saturates because this close to the incoupling point, light from the coupling objective is picked up directly. (d-f) Details of the measurement at position " $A$ " at $\omega=0.2635$, showing the interference amplitude (d) and the underlaying interference fringes (e and f).

tion. At $\omega=0.2635$ the elongation was still moderate, but the measurement at $\omega=0.2603$ shows significantly broader pulses (48(3) $\mu \mathrm{m}$ FWHM) than at higher optical frequencies. A clear beating pattern is visible in the measurement at $\omega=0.2635$. Most probably, an unwanted TM polarized mode was generated while coupling light to the structure, which "quasi-interfers" with the TE mode, resulting in the modulated amplitude pattern. Note that due to the mixing of polarizations in the near-field probe, quasi-interference can occur between orthogonally polarized modes [30, 24]. We see that the pulses are broadened, as we approached the slow-light region of the dispersion relation. From the SID measurements, the temporal shape of the pulse can not be recovered. Therefore we conducted additional experiments, to determine the pulse dispersion in a more direct fashion.

We measured the cross-correlation function of the pulses in the reference and signal branch in a PXC measurement. These interferograms are measured at six positions along the waveguide. The positions are equally spaced along the propagation direction and the first and last positions ("A" and "F") are at $10 \mu \mathrm{m}$ and $116 \mu \mathrm{m}$ distance from the waveguide input facet, respectively. The position of the fiber probe at each of these locations is highlighted with crosses in Fig. 4. The interferograms resulting from the PXC measurements are shown in Fig. 5(a-c). For clarity only the amplitude of the interferograms is presented, while all the underlaying fringes are also measured. The underlaying fringes of the measurement at position "F" in Fig. 5(a) are shown in Figs. 5(e) and 5(f). The interference fringes are well resolved. By Fourier transformation, the optical carrier frequency is recovered. We find frequencies of $\omega=0.2664(19), 0.2635(12)$ and $0.2603(8)$. The error in these values is given by the FWHM of the corresponding Fourier transforms.

Six interferograms at $\omega=0.2664$ are shown in Fig. 5(a). The interferograms are found at

\#9936 - \$15.00 USD

(C) 2006 OSA 
different delay times, corresponding to the travelling time of the pulse. We analyzed both the center of mass (CoM) and the maxima of the interferograms as a function of probe position. We found a linear dependence of the found delay time $\tau$ as a function of probe position $z$. The slope represents the group velocity. Due to the asymmetric pulse dispersion (especially at low $\omega)$, the found velocity is slightly different, depending on with method was used. We found that, the CoM-approach results in a too low $v_{g}$, whereas analyzing the maxima results in a too high group velocity. Therefore, we used both approaches to determine the group velocity, were the results represent the upper and lower error margin. In this way, we find a group velocity of $c \cdot 0.188(5)$ for $\omega=0.2664$. Similarly, the group velocities of the other measurements are also calculated. The group velocities for the measurements at $\omega=0.2635$ and 0.2603 are $c \cdot 0.151(5)$ and $c \cdot 0.116(9)$, respectively.

In addition to a difference in group velocity, we observe a broadening of the interferograms. At $\omega=0.2664$, the interferogram retains its initial shape, but as the pulses travel further in the $\mathrm{PhCW}$, the interferogram is broadened up to $0.57(5) \mathrm{ps}$ at position " $\mathrm{F}$ ". This broadening is still moderate at this high frequency, but as the frequency is reduced to $\omega=0.2603$ the pulses experience a much stronger broadening. At the same spatial position ("F"), we measured a FWHM of the interferogram of 2.2(2) ps. Interestingly, as the pulse has propagated through 106 $\mu \mathrm{m}$, the shape of the pulse has become asymmetric for all frequencies. The largest asymmetry is found in the measurements at $\omega=0.2603$.

Also other modes can be recognized in Fig. 5. Both air-guided light and a TM-polarized mode is detected at $\omega=0.2664$. These modes are visible as smaller side-lobes on the main pulse. We could discriminate these modes by their group velocities found in Fig. 5 and their spatial profile in the measurements of Fig. 4. Particularly at the measurement position at $10 \mu \mathrm{m}$ from the facet, the air-guided light is very strong. This is not surprising, since we couple light into the structure via an objective, that focusses light onto the facet. Since a diffraction limited spot at these wavelength is larger in diameter $(3 \mu \mathrm{m})$ than the membrane thickness, such coupling results in some light skimming over the surface of the PhCW.

At $\omega=0.2635$, a TM-polarized mode is very strong and causes a quasi-interference with the dominant TE-polarized mode. Specifically, a TM-polarized crystal mode travels at roughly the same group velocity as the TE defect mode. This causes the irregular pulse shapes in the interferograms in the measurement at $\omega=0.2635$. The TM-crystal mode is particularly strong in the measurements at this frequency. In the other measurements, it's influence is negligible. From a Fourier transform of the complex fields in Fig. 4, we know that multiple $k$-vectors are present in the measurement [24]. These correspond to the even TE-mode, a TM waveguide mode and a TM crystal mode, each having a different group velocity. The spatial overlap of the orthogonally polarized pulses is confirmed by selective Fourier filtering of the individual wavevectors corresponding to a single mode [32].

In Fig. 6, we have summarized our experimental findings, and compared these to the values derived from the calculated dispersion relation. In this figure, two additional experiments were added. The wavevectors are determined by means of a Fourier Transform of the SID measurements [19, 24]. With $\omega$ and $k_{z}$ known, a portion of the dispersion relation can be drawn. In Fig. 6(a), the measured dispersion relation is slightly offset $(0.5 \%$ of $\omega)$ with respect to the theoretical dispersion relation. This can be attributed to a slight offset of the theoretical dispersion relation of $\Delta \omega \approx 0.006$, compared tot the found results in the measurements. This small difference can easily be explained by assuming that the actual geometric and optical properties of the sample are slightly different from the parameters used in the simulations.

The group velocities are shown in Fig. 6(b) as a function of frequency. We find group velocities ranging from $0.188(5) \cdot c$ down to $0.108(9) \cdot c$. The qualitative agreement between experimental data and the theoretic dispersion relation is clear. Again, the measured points are

\#9936 - \$15.00 USD

(C) 2006 OSA
Received 13 December 2005; revised 13 February 2005; accepted 13 February 2006 20 February 2006 / Vol. 14, No. 4 / OPTICS EXPRESS 1668 

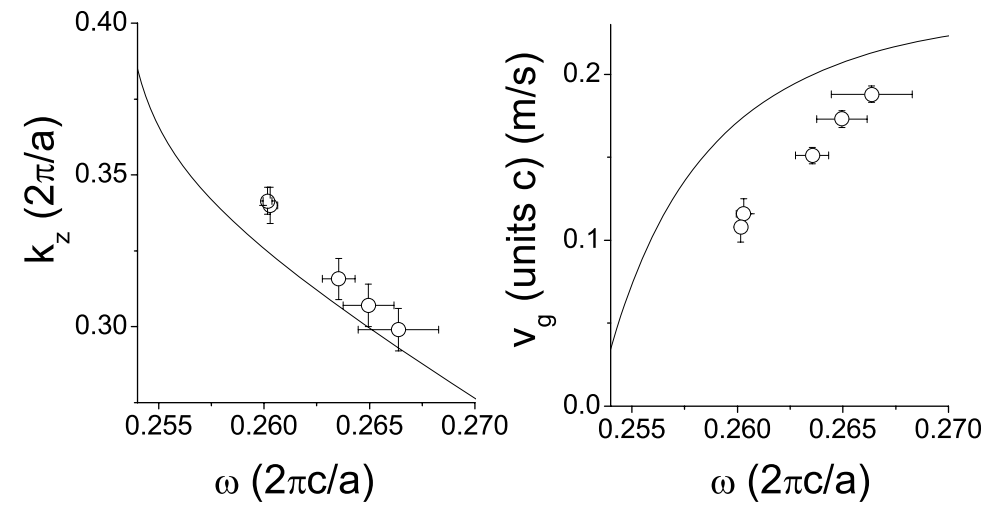

Fig. 6. Summary of the found experimental results. The carrier frequency of the pulses is found by Fourier Transformation of the interferograms. In both figures, the theoretical values are depicted by a straight line. (a) The theoretical dispersion curve and the experimentally found dispersion relation of the TE mode. The values of $k_{z}$ are found by Fourier Transformation of the data from the SID measurements in Fig. 4. (b) Group velocity of the pulses, found by evaluating the center-of-mass of the interferograms in Fig. 5.

offset in frequency with respect to the theoretic curve. The discrepancy in Fig. 6(b) (2\% of $\omega$ ) is larger than in Fig. 6(a). This suggests that the actual dispersion relation differs from the simulated curve in two aspects. First, the actual curve is offset in frequency as is observed in Fig. 6(a). Second, the actual curve runs steeper than the simulated curve, due to the difference in group velocity.

\subsection{The effect of higher-order dispersion}

In order to demonstrate the effect of higher-order dispersion on pulse propagation, we will compare our theoretical findings with the results obtained from simulations. We have solved Eq. (11) numerically using the full dispersion relation in Fig. 2(a). The numerical result describes the interferogram at a specific point on the waveguide. In this way, we can compare the results in Fig. 5(a-c) directly with what is expected from the dispersion relation. Since the found experimental dispersion relation, is not an exact match to the calculated dispersion relation, we matched the group velocities of experiment and simulation. We found that the calculated results at frequencies $\omega=0.2620,0.2585$ and 0.2565 correspond to the experimental frequencies $\omega=0.2664,0.2535$ and 0.2503 . We have calculated six interferograms at positions from $10 \mu \mathrm{m}$ up to $116 \mu \mathrm{m}$ and the results are shown in Fig. 7. Again, only the amplitude of the interferograms are shown for clarity.

In Fig. 7, the interferogram envelope hardly changes at frequency $\omega=0.2620$. At this frequency, only a slight symmetric broadening is visible after $116 \mu \mathrm{m}$ of propagation. This in contrast to the results at $\omega=0.2585$. Here, the interferograms are clearly broadened when propagating through the waveguide. This effect is present much stronger when calculating the envelopes at $\omega=0.2565$. Now, the dispersion is very strong and after $116 \mu \mathrm{m}$, the pulse are approximately 3 times longer (FWHM) than initially. At this frequency, the asymmetry of the interferogram becomes clear. Since the spectrum of the pulses is symmetric along the carrier wavelength, the asymmetry in temporal pulse shape (and also in the interferogram) can only be caused by the enhanced higher-order dispersion in the waveguide. By comparing Figs. 5 and 7 ,

\#9936 - \$15.00 USD

(C) 2006 OSA 


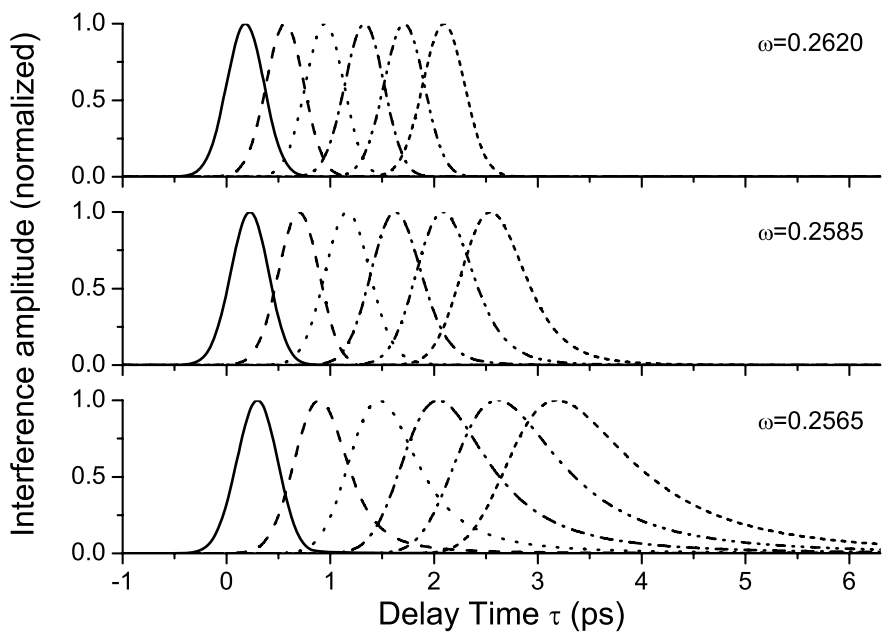

Fig. 7. Calculated amplitude of the interferograms at frequencies $0.2620,0.2585$ and 0.2565 , using the dispersion relation from the simulations (Fig. 3(a)). For each frequency, the interferogram is calculated for 6 waveguide lengths, equidistantly spaced between 10 and $116 \mu \mathrm{m}$.

we can conclude that the overall interference envelope change is similar in the measurements and the calculation.

From the theoretical interferograms corresponding to the measurements at $\omega=0.2603$, we calculated the magnitude of the dispersive terms. We found the following GVD: $\beta_{2}=-1.1(3)$. $10^{6} \mathrm{ps}^{2} / \mathrm{km}$. For TOD we find $\beta_{3}=1.1(4) \cdot 10^{5} \mathrm{ps}^{3} / \mathrm{km}$ and for the fourth order dispersion we found $\beta_{4}=-8(4) \cdot 10^{3} \mathrm{ps}^{4} / \mathrm{km}$.

Next we will demonstrate the influence of the higher-order dispersive terms, if the dispersion is approximated by the Taylor-expansion in Eq. (4). We recalculated the interferogram of Fig. $7(c)$ (position " $F$ "), since this interferogram shows the strongest effect of higher-order dispersion. First, the dispersion free propagation of light was considered, in other words $\beta_{>2}=0$ in Eq. (4) and using this dispersion, the interferogram was recalculated. In steps, the higher-order dispersive terms were added to the calculation. First only GVD and then TOD was added to the calculation. The results are shown in Fig. 8, where they are compared to the calculation using the full dispersion relation, with all dispersive orders included. Clearly, if we compare the dispersion-free propagating pulse with the full calculation, it is clear that the pulse broadens significantly and we obtain only $25 \%$ overlap between the two curves. This broadening is also visible if the GVD is included in the calculation. Now the overlap increased to 64\%. However, the shape of the interferogram is still symmetric. We obtain an asymmetric interferogram if TOD is considered. This approximates the actual interferogram quite well with $88 \%$ overlap. If also the fourth order dispersion is included (not shown), a slightly better overlap is found up to $89 \%$. Note however, that the group velocity in this calculation is still quite high $\left(v_{g}=0.116 \cdot c\right)$. If the group velocity is further decreased, higher-order dispersive effect play an increasingly important role and then, using a Taylor expansion as in Eq. (4) is an inaccurate approximation to the dispersion relation if only lower order dispersive terms are considered. 


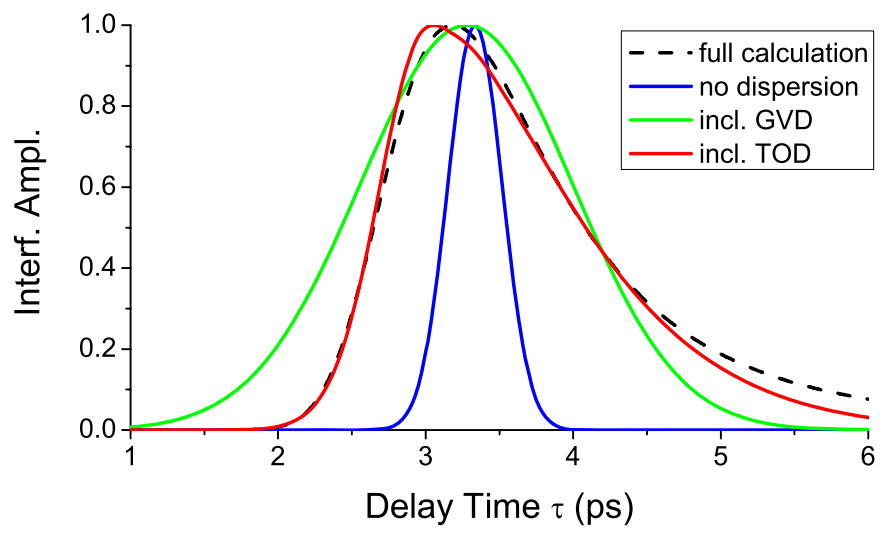

Fig. 8. The effect of higher-order dispersion on the propagation of a slow pulse. The pulse shape is calculated after $116 \mu \mathrm{m}$ of propagation at $\omega=0.2565$. The blue curve represents the interferogram of dispersion-free propagating pulses. In the green curve, the effect of GVD is included. The third order dispersion is included in the red curve. In the dashed black curve, the full dispersion relation is included.

\section{Conclusion}

We have studied the dispersion of ultrafast pulses in a photonic crystal waveguide in both experiment and theory. We locally probed the light in the waveguide with an near-field technique and monitored the pulse shapes with an interferometer. At several waveguide positions, the shape of the obtained interferogram in time was analyzed. For ultrafast pulses, we find that as the propagation length increases, the envelope of the measured interferogram becomes asymmetrically. We have modelled the interferometric measurements for comparison between experiment and theory. Our model shows that the shape of the interferogram is a direct measure for the shape of the pulse envelope in the photonic crystal waveguide, and therefore the pulses in the waveguide must also be asymmetric in time. We find that the increased higher-order dispersion has a large effect on the pulse asymmetry, even at relatively high group velocities. Depending on the incident optical frequency, the group velocity varied between $0.188(5) \cdot c$ and $0.108(9) \cdot c$. Though these group velocities are moderately low, the found values for GVD and TOD are already substantial. We have quantified the GVD from our measurements, and found anomalous dispersion up to $\beta_{2}=-1.1(3) \cdot 10^{6} \mathrm{ps}^{2} / \mathrm{km}$. Similarly, the TOD is found to be very large: values up to $\beta_{3}=1.1(4) \cdot 10^{5} \mathrm{ps}^{3} / \mathrm{km}$ are found. These parameters are sufficient to simulate the dispersive effects of a femtosecond pulse travelling through our photonic crystal waveguide at a moderate speed of $0.116 \cdot c$. As the group velocity reduces further, even higher-order dispersive terms will start to play a role. Especially at these lower optical frequencies the common approach, to approximate the dispersive properties by a Taylor expansion, is only valid if many orders of the expansion are included. We have found that the effect of higher-order dispersion in a typical photonic crystal (waveguide) strongly increases when the group velocity decreases.

Slow light devices could find their application in optical data processing. Delay times in the picosecond or nanosecond regime would be useful. In any slow-light application a trade-off between bandwidth and device size has to be found: device sizes can be reduced by exploiting slower light, but usually at the expense of large dispersion and hence a reduction of the useful bandwidth. If the studied W1 waveguide were to be used for the delaying of a 1 ps pulse by 
1 ns with respect to air, the pulse would be elongated (mainly by GVD) to $0.16 \mathrm{~ns}$, if a relatively high group velocity would be chosen $(c / 10)$. However, if one exploits a lower group velocity, for example $c / 50$ to achieve the same delay, the elongation would be up to 4 times larger (approximately $0.5 \mathrm{~ns}$ ) and the pulse shape would become asymmetric, due to the increased higher-order dispersion. In this respect, the large higher-order dispersion in the studied waveguide makes the simple W1 geometry an unlikely candidate for slow-light applications. However, in photonic crystals, one has the freedom to selectively alter the dispersive properties by changing the geometry of the lattice, or even by using combinations of lattices. This freedom will need to be used to create large-bandwidth slow-light applications that do not suffer from higher-order dispersion.

\section{Acknowledgements}

This work is part of the research program of the "Stichting voor Fundamenteel Onderzoek der Materie (FOM)", which is financially supported by the "Nederlandse organisatie voor Wetenschappelijk Onderzoek (NWO)".

\#9936 - \$15.00 USD

(C) 2006 OSA
Received 13 December 2005; revised 13 February 2005; accepted 13 February 2006 20 February 2006 / Vol. 14, No. 4 / OPTICS EXPRESS 1672 